\title{
Les infirmières indiennes émigrées dans les pays $d u$ Golfe : de l'opportunité à la stratégie
}

\section{Marie Percot}

\section{(2) OpenEdition}

1 Journals

\section{Édition électronique}

URL : https://journals.openedition.org/remi/2340

DOI : $10.4000 /$ remi.2340

ISSN : $1777-5418$

Éditeur

Université de Poitiers

\section{Édition imprimée}

Date de publication : 29 mai 2005

Pagination : 29-54

ISBN : 2-911627-39-3

ISSN : 0765-0752

\section{Référence électronique}

Marie Percot, "Les infirmières indiennes émigrées dans les pays du Golfe : de l'opportunité à la stratégie », Revue européenne des migrations internationales [En ligne], vol. $21-n^{\circ} 1$ | 2005, mis en ligne le 02 septembre 2008, consulté le 15 avril 2022. URL : http://journals.openedition.org/remi/2340 ; DOI : https://doi.org/10.4000/remi.2340

Ce document a été généré automatiquement le 15 avril 2022.

(c) Université de Poitiers 


\title{
Les infirmières indiennes émigrées dans les pays du Golfe : de l'opportunité à la stratégie
}

\author{
Marie Percot
}

1 Depuis bientôt deux générations des infirmières indiennes émigrent à l'étranger, jusqu'à présent dans leur immense majorité pour les pays du Golfe où la demande n'a pas cessé depuis la fin des années 1970. Ces toutes dernières années, de nombreux pays occidentaux (en particulier les USA et la Grande-Bretagne) ont aussi lancé des recrutements massifs d'infirmières en Inde, réorientant ainsi - au moins en partie les possibilités de parcours migratoires de ces femmes. Cet article ${ }^{1}$ vise à retracer l'histoire et l'évolution qu'a connues en trente ans cette migration dans le Golfe et à en décrire les particularités sociales. Il est aussi une première approche, sur ce terrain spécifique, des changements familiaux ou sociaux induits par la migration de ces Indiennes qualifiées qui n'est pas motivée par une absolue nécessité financière, mais essentiellement par une volonté familiale d'ascension sociale et d'accès accru aux biens de consommation. Je tenterai de montrer de quelle manière, et à leur insu, cette motivation première des familles qui soutiennent, voire initient le départ de leurs filles ou belles-filles, aboutit à faire naître chez ces dernières des aspirations à plus d'autonomie et plus d'individualisme incompatibles avec les valeurs sociales traditionnelles, sans pour autant les remettre en cause radicalement ou tout au moins de manière frontale. C'est ainsi que le retour définitif au pays s'avère souvent problématique pour ces femmes.

\section{Le contexte}

2 Les infrastructures sanitaires dans les pays du Golfe sont contemporaines de l'ensemble des infrastructures qui furent développées à la suite des dernières indépendances dans cette région et du boom pétrolier de 1973. Le premier véritable hôpital de Mascate, capitale d'Oman, a ainsi ouvert en 1978, à peine deux à trois ans plus tôt dans les 
Émirats Arabes Unis (Abu Dhabi, Dubaï). Jusqu'alors, les habitants n'avaient comme seul recours pour se faire soigner que les quelques petits hôpitaux plutôt type dispensaire des missions chrétiennes ou - pour ceux qui y avaient accès - les dispensaires des compagnies pétrolières.

3 Le personnel qui fut engagé dans les hôpitaux nouvellement construits était et reste très largement constitué d'immigrés. Si les médecins sont de nationalités très diverses (Palestiniens, Jordaniens, Anglais, Indiens, Pakistanais, etc.) et de plus en plus indigènes, en ce qui concerne les infirmières, c'est en revanche l'Inde qui constitue depuis le début le principal réservoir de main-d'œuvre ${ }^{2}$. Malgré les diverses politiques d'indigénisation de la main-d'œuvre (dites «émiratisation », « omanisation »...), la plupart des pays du Golfe n'ont toujours pas institué de formation d'infirmières conséquente $^{3}$ et devront donc faire encore appel pour des années à des femmes étrangères. Compte tenu du développement de ses infrastructures sanitaires et de l'augmentation très forte en trente ans de la population (autochtone et immigrée confondue), le Golfe représente ainsi une véritable filière pour les femmes des pays « exportateurs » : entre 40000 et 50000 Indiennes y travailleraient aujourd'hui comme infirmières ${ }^{4}$.

4 En Inde, ce métier est donc quasiment devenu synonyme de passeport pour l'émigration. La formation des infirmières est, par conséquent, devenu un marché florissant qui attire même aujourd'hui des investisseurs étrangers, un marché encouragé par un gouvernement indien qui compte explicitement sur les dividendes de cette " exportation ", de la même manière qu'il compte sur celle des informaticiens... Plus de trente mille nouvelles diplômées sortent chaque année des sept cents écoles du pays ${ }^{5}$; presque toutes ont dès le départ choisi le métier pour pouvoir travailler au moins un certain temps à l'étranger. La grande majorité de ces femmes est originaire d'un petit État du sud de l'Inde, le Kerala.

\section{Les infirmières indiennes : une spécialité du kerala}

Avec 31,8 millions d'habitants au dernier recensement ${ }^{6}$, le Kerala représente $3,1 \%$ de la population indienne. Pourtant sur les 3 à 4 millions d'Indiens émigrés dans le Golfe, environ 1,3 millions sont des Malayalis ${ }^{7}$ (Zachariah et al, $2002: 17$; Sekher, $1997: 24$ ) qui y occupent des postes à tous les niveaux de la hiérarchie du travail, même si la maind'œuvre peu ou pas qualifiée représente une large majorité de ces migrants. Je ne pourrai ici développer toutes les raisons de l'émigration massive des Malayalis et renverrai donc à la littérature qui en traite spécifiquement (voir par exemple Sekher, 1997 ; Prakash, 1994), mais j'en résumerai brièvement les causes principales.

6 La majorité des habitants du Kerala est hindoue, mais on y trouve aussi deux fortes minorités : près de $20 \%$ de musulmans et $20 \%$ de chrétiens. Ce petit État a une place très particulière en Inde, son histoire et son développement contemporains faisant figure de modèle sur de nombreux points : le taux d'alphabétisation y est de $90,9 \%$ contre $64,8 \%$ pour l'Inde entière ${ }^{8}$; le taux de natalité de 1,7 enfants par femme contre 3,5 pour l'Inde ; la durée de vie y est de 68,8 ans pour les hommes contre 59 ans pour l'Inde et de 74,4 ans pour les femmes contre 59,4 pour l'Inde entière ${ }^{9}$ (Tarabout, $1997: 253$; Théau et Venier, $2001: 24)$. En 1957, le Kerala fut le premier État au monde à élire un gouvernement communiste, le syndicalisme y reste très actif et la politique continue à mobiliser l'ensemble de la société (Jeffrey, 1993 : 126-140). Ce qui retient 
aussi largement l'attention des observateurs, c'est le taux élevé d'alphabétisation des femmes (87,7\% des femmes du Kerala sont alphabétisées contre 53,6 \% pour l'ensemble des Indiennes), leur niveau d'éducation et leur place dans le monde du travail. De nombreux chercheurs (cf. par exemple Jeffrey, 1993; Saradamoni, 1999; Théau et Venier, 2001) attribuent en partie cette spécificité du Kerala à un héritage de la tradition matrilinéaire qui a prévalu dans cette région jusqu'au début du $\mathrm{xx}^{\mathrm{e}}$ siècle. En revanche, le développement économique n'a pas suivi dans les mêmes proportions, en raison notamment de la faiblesse des investissements privés. Les entrepreneurs indiens ou étrangers pointent systématiquement le "handicap » d'une main-d'œuvre locale trop revendicative, demandant des salaires plus élevés qu'ailleurs en Inde et trop rapidement prête à l'action syndicale... Depuis plus de trente ans, les habitants sont ainsi contraints pour beaucoup à l'émigration. Les émigrants malayalis, estimés actuellement à plus de deux millions, partent travailler dans d'autres États de l'Inde et, majoritairement, dans les pays du Golfe; jusqu'à présent une petite proportion seulement (2\%) émigre vers l'Occident (Zachariah et al, 1999: 9) ${ }^{10}$. L'argent envoyé au pays par les émigrés à l'étranger est désormais la seconde ressource de cet État après l'exportation des épices et du caoutchouc (Zachariah et al, 2000 : 21). "Gulf money ", précise-t-on au Kerala lorsque l'on passe devant une belle maison, un magasin flambant neuf ou lorsque l'on croise une voiture de luxe.

7 Une autre caractéristique du Kerala est l'importance qu'y représente la minorité chrétienne (près de $20 \%$ contre 2,4\% pour l'Inde entière). Dans certains districts comme celui de Kottayam, les Chrétiens forment même la majorité de la population. La communauté chrétienne du Kerala a pour particularité de n'être pas seulement une conséquence de la colonisation et des conversions qu'elle a pu entraîner. Le christianisme y était implanté bien avant: les chrétiens «syrians» du Kerala font même remonter leur adoption de cette religion à l'évangélisation de leur pays par l'apôtre Thomas lui-même au I ${ }^{\mathrm{er}}$ siècle de notre ère (Visvanathan, 1993 : VII-XIII) ${ }^{11}$. Quoi qu'il en soit, dès le vII ${ }^{\mathrm{e}}$ siècle, la présence de chrétiens au Kerala est attestée par les voyageurs arabes qui fréquentent ses côtes.

Or, c'est cette communauté chrétienne du Kerala ${ }^{12}$ qui fournit plus de $90 \%$ des infirmières de l'État et une majorité des infirmières indiennes en général (Mohan, 1990 : 16). Cela s'explique assez aisément dans le contexte d'un pays majoritairement hindou. Deux notions clés de l'hindouisme sont celles de pureté et de pollution (Dumont, 1966) : le métier d'infirmière parce qu'il implique un contact permanent à la souillure (plaies, excréments, vomissures, etc.) est considéré comme particulièrement impur. Il ne saurait toutefois relever des intouchables puisqu'un hindou de caste ne peut pas - selon l'orthodoxie - être touché par un intouchable et, par conséquent, être soigné par lui. Le fait que des femmes chrétiennes occupent cette fonction résout en quelque sorte la question. Pour les chrétiens, en revanche, ce métier, s'il est encore loin d'être prestigieux, renvoie à des valeurs traditionnelles de leur foi, d'autant que les premières infirmières ont été des religieuses - missionnaires tout d'abord, puis indiennes $-{ }^{13}$. De surcroît, les Chrétiens du Kerala n'ont pas (ou n'ont plus) de réelles réticences à voir leurs femmes exercer un travail à l'extérieur du foyer, réticence qui persiste dans d'autres communautés comme les Hindous et surtout les Musulmans ${ }^{14}$.

En Inde, les infirmières sont ainsi devenues une «spécialité » du Kerala. On constate d'ailleurs que plus d'un tiers des écoles formant au métier y sont situées et que les Malayalies forment la majorité des effectifs des écoles d'infirmières des autres États du 
pays. Les écoles du Kerala étant saturées de demandes, et donc très sélectives, les candidates n'hésitent pas à partir étudier à des milliers de kilomètres de chez elles : une première coupure d'avec le milieu familial et une nécessaire prise d'indépendance ou au moins d'autonomie qui a sans nul doute son importance dans leur aptitude à émigrer ensuite seules à l'étranger. D'après mes propres observations, plus de $90 \%$ des infirmières indiennes émigrées dans les pays du Golfe sont des Chrétiennes originaires du Kerala. Elles représentent donc une bonne partie des femmes malayalies présentes dans le Golfe puisque, selon les estimations, sur les 1,3 millions d'émigrés malayalis, $10 \%$ seulement sont des femmes (Zachariah et al., $2002: 47$ ).

\section{Des femmes de milieu rural}

Sur les deux cents infirmières ou étudiantes infirmières que j'ai rencontrées près de $90 \%$ ont décrit leur père comme "fermier" possédant 2 à 3 acres $^{15}$ de cultures commerciales (hévéa, épices, bananiers...). Au Kerala, de tels fermiers ne sont pas vraiment agriculteurs; ils contribuent certes à l'exploitation agricole, mais ils disposent d'un ou deux ouvriers pour faire l'essentiel du travail. Nombre de ces fermiers ont parallèlement un travail salarié (un emploi de bureau en général). La mère est presque toujours décrite comme femme au foyer, parfois aidée d'une servante. Une trentaine seulement des infirmières ou étudiantes interrogées avaient des parents diplômés, travaillant dans l'administration comme comptables, employés d'écriture ou techniciens ; quinze d'entre elles avaient des mères institutrices. La maison des familles d'étudiantes, où j'ai été invitée, est bien équipée (réfrigérateur - que seule $13 \%$ de la population malayalie possède - télévision, téléphone, motocyclette...). Elle est caractéristique du niveau de vie de la classe moyenne rurale ${ }^{16}$. Mais la plupart des parents que j'ai rencontrés ne parlaient pas ou peu anglais, ce qui - en Inde - indique un niveau d'étude assez bas. L'essentiel des infirmières représente donc la première génération dans leur famille à poursuivre des études au-delà de l'école secondaire ${ }^{17}$ (le diplôme se préparant en trois ans après l'obtention de l'équivalent du baccalauréat dans des écoles où l'enseignement se fait exclusivement en anglais).

11 Malgré des revenus décents selon les standards indiens, l'investissement dans les études des enfants représente pour les parents un gros effort financier, notamment pour celles d'infirmière. En effet, il y a une vingtaine d'années, la plupart des étudiantes étaient formées dans les écoles publiques qui sont gratuites et assuraient même un revenu dès la seconde année. Mais depuis que la filière du Golfe (et a fortiori la filière occidentale) s'est développée, les écoles publiques ne suffisent plus au grand nombre de candidates. Elles sont aussi devenues d'autant plus sélectives qu'elles restent la voie royale pour intégrer les hôpitaux gouvernementaux indiens et donc le fonctionnariat avec tous ses avantages (pour celles qui ne pourraient émigrer ou qui se trouvent entre deux périodes de migration). La majorité des étudiantes doivent désormais s'inscrire dans les nombreuses écoles privées qui se sont ouvertes pour répondre à la demande et dont le coût se situe entre 15000 et 30000 Roupies ${ }^{18}$ par an, auxquelles il faut ajouter les frais de logement et de nourriture lorsque l'école est loin du domicile parental; à titre de comparaison, une infirmière débutante gagne 2500 Roupies par mois, un instituteur du secteur public 3000 Roupies. Le financement des études oblige souvent les parents à souscrire un emprunt (ordinairement auprès d'autres membres de la famille, parfois auprès d'une banque). Il est, par conséquent, 
assez rare que le choix du métier d'infirmière soit le fruit d'une décision individuelle de la jeune fille; il s'agit plutôt d'une stratégie familiale où les parents attendent un retour sur investissement.

12 Un retour qui se fera par l'aide financière ou les cadeaux que leur fille émigrée devra leur envoyer, au moins tant qu'elle sera célibataire, mais retour qui se fera surtout au moment de son mariage : la règle - en Inde - étant le mariage arrangé avec une dot à la charge des parents de la femme (quelle que soit la religion ou la caste de la famille). La dot (streedhanam en sanscrit) représente théoriquement la part d'héritage d'une fille que le père donne au moment du mariage de celle-ci ; elle est constituée de bijoux en or, d'argent liquide, de divers biens de consommation et parfois de biens fonciers; de fait, si la jeune épouse garde une part de contrôle relative sur les bijoux et, au Kerala au moins, sur les biens fonciers qui restent enregistrés à son nom, la jouissance de l'argent ou des biens de consommation revient à la belle-famille ${ }^{19}$. Bien qu'interdite légalement depuis 1961, la dot reste la norme partout en Inde et elle atteint au Kerala des sommes importantes, particulièrement chez les Chrétiens. Il y va du prestige de la famille et rares sont ceux qui cherchent à s'y soustraire (ceux qui le font sont presque tous des militants « anti-dot » affichés qui en soulignent les conséquences sociales désastreuses et, dans les annonces matrimoniales, sont presque toujours des parents de garçons à marier puisqu'on ne peut les soupçonner

- contrairement aux parents de filles - d'agir par intérêt). Un mariage où j'ai été invitée fournit un exemple du coût de la dot. Le père de la mariée, un mécanicien qui gagne 5000 roupies par mois avait offert 200000 Roupies de dot (en or et en espèces) à la famille du marié. Pour réunir l'argent nécessaire, il avait vendu les quelques arpents de terre agricole lui venant de ses propres parents et fait appel à un frère émigré pour compléter la somme promise. Pour le mariage de leur seconde fille (qui comme la première n'avait pas pu poursuivre des études), les parents comptaient sur les économies de leur fils pour peu qu'il réussisse à partir dans le Golfe. La surenchère sur les dots est un phénomène que l'on retrouve dans l'Inde entière et qui s'explique en grande partie par le développement du consumérisme depuis une vingtaine d'années (Bruneï, 1996). Dans le cas du Kerala, l'argent provenant d'une émigration massive contribue sans nul doute à la surenchère sur les dots.

Or, sur le marché matrimonial, un diplôme pour un métier qui ne connaît pas de chômage est en soi une valeur qui permet à la famille d'une fille de faire baisser la somme offerte au moment même du mariage puisque c'est la belle-famille de la mariée qui profitera ensuite des revenus de leur nouvelle bru (la résidence du jeune couple étant patrilocale et les revenus d'une jeune femme devant traditionnellement bénéficier à sa belle-famille et non à sa propre famille). Dans le cas des infirmières, c'est non seulement un salaire supplémentaire qui revient ainsi à la belle-famille, mais un moyen d'émigrer pour le conjoint et, à terme, pour d'autres membres de sa famille: une opportunité qui est toujours prise en compte dans le calcul de la dot et permet, en particulier, de baisser grandement la somme offerte en cash.

14 C'est ainsi que malgré le traditionnel manque de prestige de leur métier, les infirmières sont devenues des partis de choix qui, comme le dit Shoba George dans une étude sur les infirmières indiennes émigrées aux États-Unis (George, 2000: 152), pour peu «qu'elles viennent d'un bon milieu familial [...] sont réservées avant même la fin de leur scolarité ». Dans les stratégies d'hypergamie qui ont cours en Inde, une fille, infirmière, est désormais devenue un atout, ainsi que l'explique l'une d'entre elles : 
15 «Il y a 20 ans, les gens n'avaient aucun respect pour nous. Ils disaient que c'était un métier de domestique. Les gens jasaient aussi parce qu'on doit toucher des hommes bien sûr et ils nous faisaient une mauvaise réputation. Quand je me suis mariée, ma belle-mère disait à mon mari que ce serait mieux que je ne travaille pas [...] Mais aujourd'hui une infirmière est toujours sûre de trouver du travail, pour elle il n'y a pas de chômage. Et surtout elle peut émigrer dans les pays arabes et même en Amérique. Alors même dans les bonnes familles maintenant on cherche des infirmières. Moi, j'ai épousé un technicien agricole, alors que ma nièce qui est infirmière à Dubaï vient de se marier avec un ingénieur qui a fait ses études à Delhi $»^{20}$.

Ou encore, Nirmal, jeune marié fraîchement débarqué à Mascate qui avoue qu'il n'aurait jamais imaginé "avoir la chance d'épouser une infirmière, surtout une infirmière travaillant dans un hôpital gouvernemental [d'un pays du Golfe] ${ }^{21}$ ", alors qu'il a "tout juste un diplôme de tourisme» : cela a probablement pu se produire parce que le jeune couple a fait un mariage dit «d'amour » et s'est donc passé de l'avis des parents de la jeune fille.

On peut aussi vérifier cet « attrait » nouveau pour les infirmières (et donc la diminution $\mathrm{du}$ stigmate social) dans de nombreuses annonces matrimoniales des journaux indiens ou sites Internet spécialisés :

« Homme, chrétien jacobite, équilibré et talentueux, 31 ans, Master d'Engineering, très actif dans l'Église, les activités politiques et culturelles locales, cherche infirmière »; " Diplômé supérieur, 32 ans, catholique romain, vivant à Singapour, cherche infirmière travaillant en Arabie Saoudite ou dans hôpital gouvernemental en Inde "; " Cherchons pour fils unique, Maîtrise en Science, 30 ans, une jeune fille au teint clair, avec un bon caractère et très croyante, âgée de 25 ans environ. Jeunes filles infirmières et travaillant au Moyen-Orient auront la préférence ${ }^{22}$.

Des préjugés subsistent certes sur les mœurs de ces jeunes femmes, particulièrement pour celles qui se trouvent seules à l'étranger. Un chauffeur de taxi de Kottayam, luimême chrétien, m'a ainsi tenu ce discours : "On se demande ce qu'elles font quand elles ont fini le travail. Parce qu'elles sont toutes seules là-bas, alors c'est facile pour elles de gagner plus en traînant dans les rues si vous voyez ce que je veux dire... ». Shoba George souligne aussi cette réputation de «femmes faciles » qui leur est encore souvent attribuée (George, 2000 : 146). Filippo et Caroline Osella notent, pour leur part, que dans le milieu hindou qu'ils ont étudié au Kerala, les gens reprochent fréquemment aux Chrétiens d'être prêts à tout pour gagner de l'argent : prêts non seulement à faire travailler leurs femmes à l'extérieur de la maison, mais même "à les envoyer aux Arabes comme infirmières » (Osella, 2000: 44) ${ }^{23}$. Pourtant, outre les propositions matrimoniales plus intéressantes socialement parlant, on observe un autre signe du recul des a priori envers le métier d'infirmière que soulignent toutes les directrices d'école : des jeunes filles hindoues (y compris de haute caste) et même des jeunes filles musulmanes commencent à s'inscrire pour cette formation depuis un peu moins de dix ans, avec les mêmes projets migratoires que leurs compatriotes chrétiennes ${ }^{24}$. Et c'est sans nul doute la possibilité d'émigration et l'argent potentiel qu'elle représente qui ont pesé pour atténuer les stigmates jusqu'alors attachés à ce métier : un salaire d'infirmière dans le Golfe peut être jusqu'à quinze fois supérieur à ce qu'il est en Inde, soit approchant les 45000 Roupies par mois, une somme très importante dans ce pays. Toutefois, un autre argument est désormais souvent cité au Kerala pour justifier la promotion du statut d'infirmière : ce serait la «prise de conscience » par la société malayalie de la technicité 
et du niveau d'éducation requis pour exercer ce métier (la représentation du métier comme étant principalement constitué de contact avec le corps du malade et donc objet de souillure potentielle s'effaçant au profit d'une représentation où priment gestes et pratiques purement techniques comme la pose de sondes complexes ou de perfusions, parfois même liées à une technologie ultra moderne, comme les injections nécessaires pour un scanner ou une IRM).

\section{Emigrer dans le golfe : une histoire d'opportunité}

Les premières infirmières indiennes ont été embauchées dans le Golfe vers la fin des années 1970. Jusqu'alors, le meilleur débouché pour une infirmière indienne était l'armée ou un poste dans un hôpital gouvernemental indien, l'un comme l'autre offrant de nombreux avantages par rapport au privé (salaire, logement, assurance-maladie, retraite). Deux options qui gardent tout leur attrait puisqu'il semblerait que celles qui ont obtenu de tels postes hésitent encore à faire le pas de l'émigration si ce n'est le temps d'un an ou deux qu'elles peuvent prendre en disponibilitée ${ }^{5}$. Mais avant le recrutement lancé par les pays du Golfe, aucune infirmière indienne n'avait imaginé pouvoir émigrer grâce à sa profession. Les premières à partir ont ainsi le sentiment d'avoir été de véritables pionnières : « Hormis le temps de mes études, je n'avais jamais vécu seule. Je n'avais jamais voyagé à l'étranger et personne de ma famille ne travaillait à l'étranger. J'étais plutôt inquiète, mais le salaire était très bon, on me promettait un logement et un billet par an pour revenir en Inde [...] Je me suis dit que je ne pouvais pas cracher sur autant d'argent ", explique Susan qui est arrivée en Oman en 1979 et y est restée depuis. L'une de ses collègues, arrivée deux ans plus tard précise : "J'ai été recrutée à Delhi où j'habitais avec ma belle-famille depuis dix ans [...] À cette époque, le recrutement était direct, on n'avait pas à passer par des agences et à payer trop cher. Quand nous sommes arrivées, tout un groupe d'infirmières, on nous attendait à la descente de l'avion. Il y avait tout un tas d'officiels, des discours, des jus de fruit... c'est tout juste s'il n'y avait pas un orchestre! On avait l'impression d'être des personnes importantes! Mais au début, ça n'était pas facile, on se sentait un peu perdues. On n'avait jamais imaginé se retrouver comme ça, toutes seules, loin de la famille. Heureusement, nous étions logées ensemble et comme nous venions presque toutes du Kerala, c'était rassurant. On s'est vite retrouvées aussi autour de la religion: on priait ensemble, on chantait des cantiques... ".

Toutes les femmes que j'ai rencontrées et qui sont arrivées à Oman ou aux Émirats dans ces années-là sont venues seules avec un contrat qui ne permettait pas le regroupement familial. Toutes cependant étaient mariées et mères de famille au moment de leur migration. Famille et belle-famille semblent pourtant avoir encouragé, voire initié, ce départ en informant par exemple leur fille, belle-sœur ou belle-fille d'une telle opportunité et, surtout, en contribuant aux frais de départ. Cette migration en célibataire des infirmières ne paraît pas avoir concrètement engendré de difficultés importantes. Les femmes indiennes, grâce aux liens toujours très forts qui existent à l'intérieur de la famille, où la cohabitation avec la belle-famille est encore monnaie courante, ont de fait plus de facilité que les femmes occidentales à trouver quelqu'un à qui confier leurs enfants lorsqu'elles travaillent à l'extérieur de la maison. Concrètement mais aussi idéologiquement, l'émigration d'une mère de famille n'est donc pas vue comme aussi problématique qu'elle le serait en Occident par exemple ${ }^{26}$. Ainsi, si toutes les infirmières expriment leur chagrin de devoir (ou d'avoir dû) se 
passer aussi longtemps de la présence de leurs enfants, se disent en revanche assurées de leur bien-être.

Les infirmières arrivées dans les premières années mettent l'accent sur deux points. Tout d'abord, sur les conditions dans lesquelles elles ont exercé leur travail dans les premiers temps: "On ne peut plus imaginer ce que c'était dans ce temps-là. La plupart des malades n'avaient jamais été à l'hôpital, ils ne savaient même pas ce que c'était. Jusqu'alors, les gens riches allaient se faire soigner en Inde ${ }^{27}$. Nos patients ne parlaient pas un mot d'anglais et ils étaient effrayés par les soins qu'on leur donnait. Je me souviens d'une femme qui a accouché pendant son transport à l'hôpital. J'étais avec elle dans l'ambulance. Il fallait lui faire une épisiotomie, mais elle refusait en hurlant. Résultat, le bébé n'a pas résisté... et ce n'est qu'un exemple ». Ensuite, sur l'aspect du pays lorsqu'elles sont arrivées : "Il n'y avait presque rien à ce moment-là. Mascate, c'était presque un village. Il n'y avait pas d'autoroutes, pas de grands immeubles, presque pas de magasins et beaucoup moins d'étrangers. Aujourd'hui, on peut faire ses courses comme si on était à Trivandrum ${ }^{28}$. D'ailleurs les Malayalis ont la plupart des commerces. Il y a des écoles indiennes, des cinémas indiens, des journaux indiens et des églises. Mais tout ça n'existait pas quand nous sommes arrivées, c'était vraiment le désert ».

Leur vie se déroulait donc en milieu fermé : l'hôpital pour le travail et les cours d'arabe, alors obligatoires, et le foyer-logement où elles se retrouvaient entre consœurs du Kerala. Pas de téléphone, pas d'Internet alors, le seul lien avec la famille était le courrier qui mettait plus de deux semaines dans un sens ou dans l'autre. Comme le dit Bindu, arrivée dans les Émirats, à Fujeirah, en 1981: «On arrivait à tenir parce qu'on comptait le temps. On avait un contrat d'un an ou deux et on se disait toutes qu'après ça, on rentrerait. Et puis comme on ne dépensait absolument rien, on était fières aussi de pouvoir envoyer autant d'argent à la famille. Moi, je gagnais dix fois plus que je ne gagnais en Inde. Je gagnais plus que mon mari et ses deux frères dans leur business. En Inde, mon salaire aidait la famille, là il permettait de faire de vrais projets ".

Ces femmes de la "première époque » de la migration indienne dans le Golfe, et qui y sont restées depuis, continuent à alimenter de leurs récits la représentation que toutes les infirmières que j'ai rencontrées se font des habitants des pays du Golfe (représentation au demeurant partagée par l'ensemble des émigrés indiens) : «Ils sont racistes envers les Indiens, ils nous considèrent comme des esclaves tout juste bons à travailler, alors qu'eux, ils sont tout à fait incultes (uneducated) et sont incapables de faire les choses par eux-mêmes. Ce sont les étrangers qui ont tout construit ici et qui continuent à tout faire marcher. S'ils n'avaient pas eu la chance d'avoir du pétrole, ils en seraient toujours à marcher derrière leurs chameaux ». "Uneducated » est le vocable systématiquement utilisé par les femmes avec lesquelles j'ai parlé. Il s'agit du terme habituellement employé en Inde quand on parle du « petit peuple » ou des pauvres et qui signifie tout à la fois inculte, fruste, sans éducation. C'est donc bien un sentiment de frustration qu'elles ressentent (et que semblent ressentir les Indiens en général) à se retrouver sous les ordres (au moins administrativement) et à la merci (légalement) de ces indigènes à la fois méprisants et méprisés. Frustration d'autant plus forte que leur statut est plus précaire : la plupart des infirmières ont un contrat d'un an renouvelable. Un contrat reconduit, sauf si, expliquent-elles, elles sont enceintes avant le temps réglementaire (généralement deux ans de travail), sauf si elles tombent malades, sauf si un chef les prend en grippe, etc. Sauf si, leurs employeurs décident d'embaucher des femmes d'autres nationalités (c'est ainsi que les Indiennes vivent la relation aux infirmières philippines en termes de concurrence) ${ }^{29}$, sauf si «l'émiratisation » se poursuit, sauf si 
un nouveau conflit comme celui du Koweit se déclare... Avec un statut légal des immigrés qui leur attribue très peu de droits, et - en particulier - une autorisation de séjour totalement liée au bon vouloir de l'employeur, toutes ces raisons réelles ou fantasmées aboutissent à créer un sentiment d'insécurité ${ }^{30}$. Ce sentiment est ressenti par toutes les infirmières, y compris par celles qui sont là depuis plus de vingt ans : «On ne peut pas considérer que l'on est chez nous ici, jamais, même après des années, même si on a tout ce qu'il faut pour vivre comme chez nous. Parce qu'on n'a aucun droit, juste celui de se taire et de travailler. Et que tout est fait pour qu'on le sente. Ce n'est pas comme en Occident, ici on ne s'installe pas. Même si on reste longtemps, on sait qu'au moins pour la retraite, on rentrera chez nous. Personne ne veut finir sa vie ici » ou "Ici on n'a pas le droit d'acheter une maison et on n'a plus de visa lorsque l'on atteint la retraite. Alors le retour ou pas au pays n'est même pas une question». Elles font aussi souvent remarquer que la nationalité est impossible à acquérir pour des Indiens, même s'ils le voulaient: «En Oman, il y a eu deux Indiens naturalisés depuis l'indépendance. Deux en tout dont un Chrétien du Kerala qui est le chirurgien esthétique du Sultan... J'ai un fils qui est né et a grandi ici, et bien maintenant il lui faut un visa pour venir nous voir!».

$\mathrm{Au}$ demeurant, les infirmières indiennes soulignent que les uniques relations qu'elles entretiennent avec les indigènes sont les relations professionnelles. Seule une femme, parmi toutes celles que j'ai interrogées, est entrée dans une maison émiratie (à l'occasion d'un mariage). Anh Nga Longva dans une étude sur le Koweit (Longva, 1997) confirme cette totale absence de relations entre autochtones et émigrés, quelle que soit leur nationalité. Une situation qui n'a sans aucun doute fait que s'affirmer dans les pays du Golfe et qui conduit à des formes de ségrégation visibles au quotidien, étant donné l'importance des communautés immigrées. Les infirmières indiennes les plus anciennement émigrées ont dû apprendre à parler, lire et écrire l'arabe dès leur arrivée et sont réellement capables de le parler. Celles qui sont arrivées depuis une dizaine d'années ne le parlent pas et elles sont peu nombreuses à suivre les cours d'arabe proposés dans les hôpitaux gouvernementaux. Il est à noter en ce qui concerne la langue, que ce sont plutôt les indigènes qui ont dû s'adapter et, comme l'explique une amie yéménite qui habite Abu Dhabi, l'arabe ne suffit plus dès lors que l'on sort de la maison: «Ici, si on ne parle pas anglais, on est perdu » (cela vaut pour tous les pays du Golfe à l'exception peut-être de l'Arabie Saoudite où, au minimum, les cours d'arabe sont toujours obligatoires pour les infirmières immigrées). Au cours de ces trente dernières années, l'émigration indienne en particulier a pris une telle importance qu'il est effectivement possible de vivre totalement "à l'indienne » dans tous les pays du Golfe (et plus spécifiquement «à la keralaise » vu le nombre de Malayalis présents dans le commerce). On y trouve, en plus des magasins (de nourriture, vêtements, bijouterie, musique, livres...), des églises ou des temples, des écoles, des cinémas ou encore des journaux indiens édités dans le Golfe. Grâce au satellite, il est aussi possible de capter la plupart des chaînes de télévision indiennes. Cependant ainsi que le disent tous les émigrés malayalis que j'ai rencontrés : on peut vivre comme chez soi à peu près partout dans le Golfe ${ }^{31}$, mais sans du tout se sentir chez soi. Phénomène classique de la migration toutefois, alors que les émigrés pensent partir juste le temps d'amasser quelques économies, le séjour se prolonge. Il en va de même pour les infirmières indiennes. La plupart sont parties avec l'idée de rester deux ou trois ans, mais il semble que le séjour moyen soit d'au moins cinq ans, parfois beaucoup plus lorsque leur mari a fini par les rejoindre. Il n'y a guère que celles - peu nombreuses - qui, pour diverses 
raisons $^{32}$, ont dû y rester seules qui soient rentrées au pays dans les temps prévus au départ.

\section{Travailler dans le golfe : une parenthèse qui dure et une maternité « à temps partiel »}

Presque toutes les infirmières, arrivées seules dans les années 1980, ont finalement réussi à faire venir mari et parfois enfants. Soit en trouvant un employeur pour leur époux, soit en le faisant venir avec un «visiting visa » ce qui lui laissait trois mois pour trouver un emploi sur place. Le mari a ensuite pu faire jouer le regroupement familial pour les enfants (dans le Golfe, seuls les hommes peuvent légalement obtenir un visa familial). Un choix que n'ont pas fait tous les couples cependant, nombre d'entre eux préférant laisser leurs enfants en Inde afin d'économiser plus. Si dans les premiers temps, ce sont les femmes qui ont ouvert les portes du Golfe à leur époux, la situation s'est ensuite diversifiée. Les infirmières qui ont migré à partir de la fin des années 1980 ne sont pas toujours arrivées seules. Le nombre de Malayalis dans le Golfe n'ayant cessé d'augmenter, beaucoup d'entre elles avaient au minimum un frère, un beau-frère, voire un père déjà sur place qui, en général, leur ont trouvé du travail. En outre des émigrés malayalis en quête d'épouse ont commencé à chercher des infirmières sur le marché matrimonial du Kerala, sachant qu'elles étaient assurées de trouver du travail avec un bon salaire. Comparativement à d'autres métiers, les infirmières sont bien payées dans le Golfe : alors qu'elles gagnent de dix à quinze fois plus qu'elles ne gagneraient en Inde, un manœuvre lui ne gagne que trois à quatre fois plus (il est à noter que dans bien des cas, le revenu des infirmières est supérieur à celui de leur époux, même si celui-ci possède une qualification au moins égale, voire supérieure et exerce dans le Golfe un métier correspondant à cette qualification). Dès lors, les infirmières n'ont plus été cantonnées dans les foyers des hôpitaux, mais ont aussi commencé à vivre "en ville ", en famille. Leur vie dans le Golfe se déroulant désormais, pour beaucoup d'entre elles, au sein de leur propre communauté et souvent entourées de leur propre famille est devenue moins spartiate, pourrait-on dire. Même les infirmières vivant dans les foyers ont maintenant l'occasion de sortir de l'hôpital pour des invitations chez des amis, des activités paroissiales, du shopping, etc. Toutefois, la vie de famille est loin d'être la règle pour les infirmières dans le Golfe. Un grand nombre de couples continuent à vivre séparément : parce que le conjoint est resté en Inde ou encore, fréquemment, parce que les conjoints n'ont pas trouvé de travail dans la même ville (voire dans le même pays) ${ }^{33}$. J'ai ainsi rencontré un homme très actif au sein d'une église catholique de Mascate dont l'épouse, infirmière, travaillait à plus de $400 \mathrm{~km}$ de là et qui m'a expliqué que cette situation est suffisamment courante pour qu'il ait monté un groupe de prière réunissant les gens concernés (plus de 150 couples rien que pour la paroisse «St-Peter and St-Paul » de Mascate).

Contrairement aux émigrés malayalis les plus pauvres qui ne s'accordent qu'un retour au pays tous les trois ans au mieux (Sekher, $1997: 24$ ), les infirmières et leurs proches effectuent régulièrement des aller-retour vers le Kerala, pour des mariages dans la famille, des funérailles ou pour contrôler les travaux de construction d'une maison. Si les infirmières font remarquer que ces voyages limitent leur capacité à épargner, elles les jugent cependant indispensables. Presque toutes par exemple vont accoucher au Kerala (respectant en cela la tradition indienne qui veut que les filles retournent chez 
leurs parents pour chaque accouchement). Celles qui vivent avec leur mari reviennent avec le nouveau-né ; celles qui vivent seules n'ont d'autre choix que de laisser l'enfant derrière elles, au soin de la belle-famille le plus souvent ${ }^{34}$. La plupart du temps, elles réussissent à prendre trois mois de congé à l'occasion de leur accouchement, mais j'ai rencontré plusieurs mères qui avaient dû repartir en laissant un bébé à peine âgé d'un mois. La séparation d'avec le nouveau-né est vécue comme un traumatisme, mais elle ne remet pas en cause le projet migratoire : «Ils me manquent, mais c'est pour eux que je le fais ", tel est le commentaire habituel. Tous les cas de figure se présentent ensuite pour les enfants de parents émigrés dans le Golfe. Quand cela est possible, c'est-à-dire si les deux parents résident ensemble et qu'ils ont trouvé un moyen pour les faire garder, les enfants petits vivent avec eux. Qu'un problème survienne - problème de logement, mutation du père ou plus souvent problème de garde, les crèches étant très chères et les employées de maison difficiles à obtenir ${ }^{35}$ - et les enfants seront renvoyés en Inde. Il est ainsi fréquent que les enfants fassent des séjours alternés d'une rive à l'autre de la mer d'Arabie. Les fratries ne sont pas non plus systématiquement réunies : les parents peuvent parfois garder auprès d'eux un enfant en âge d'aller à l'école mais pas un nourrisson ou, inversement, un nourrisson mais pas un enfant d'âge scolaire s'il n'y a pas d'école indienne à proximité par exemple. Toutefois dès qu'ils atteignent l'adolescence, les enfants retournent en Inde pour poursuivre leurs études, souvent dans des internats réputés, des "boarding schools", véritable héritage de la colonisation britannique où l'enseignement se fait en anglais. Cette stratégie est adoptée par presque toutes les familles indiennes du Golfe et n'est pas ici liée au travail de la mère. Les raisons avancées par mes interlocutrices sont de deux ordres: tout d'abord, expliquent-elles, parce que les bons collèges (i.e. où

- au minimum - on enseigne en anglais) sont trop chers dans le Golfe, ensuite parce qu'il est bon que les enfants « reprennent pied » dans leur pays d'origine où de toute façon ils devront retourner pour leurs études supérieures puisque les universités du Golfe sont considérées par les Indiens émigrés comme financièrement inabordables et surtout comme sans valeur. "Est-ce que quelqu'un a déjà entendu parler de l'université de Mascate ou d'Abu Dhabi? Alors que les universités indiennes, elles, ont une bonne réputation ", précise Jenny, une infirmière dont le fils étudie à Bangalore dans une école créée par Microsoft et dont la fille termine une maîtrise de sociologie à l'université de Delhi avec le projet de poursuivre par un doctorat aux États-Unis. Au total, cette femme aura vécu six ans avec son fils de 20 ans et neuf ans avec sa fille de 22 ans. Les infirmières émigrées se retrouvent donc fréquemment en situation de "mères à temps partiel ", selon leur propre expression, une situation qu'elles vivent pourtant comme un moindre mal : «On n'a rien à attendre en restant au Kerala. Si on a un peu d'ambition, il vaut mieux partir».

28 Toutes aussi soulignent la particularité de l'émigration dans le Golfe par rapport à l'émigration en Occident : «Ici, on travaille et on économise avec l'idée de rentrer chez nous un jour pour vivre enfin notre vie. En Occident, c'est différent, parce qu'on peut s'y installer vraiment si on veut. Les enfants peuvent y grandir, on peut prendre la nationalité, on peut acheter une maison, se faire une nouvelle vie. Le danger, c'est que les enfants oublient leur pays d'origine et ça, ça n'est pas forcément bon pour la famille». Justification, contradiction? Quoi qu'il en soit, le séjour forcément limité dans le temps de la migration dans le Golfe est ce à quoi se raccrochent celles qui $\mathrm{y}$ travaillent depuis déjà plusieurs années: l'émigration est ainsi vue comme une parenthèse qui leur permettra de revenir s'installer au Kerala en ayant nettement 
progressé dans l'échelle sociale sans pour autant risquer "d'abandonner leur pays » (conséquence probable, selon elles, d'une migration en Occident). Plusieurs directrices d'école d'infirmières au Kerala m'ont au demeurant fait remarquer que les parents poussent plutôt leurs filles à émigrer vers les pays du Golfe : «Même si les salaires y sont moins bons qu'en Occident, ils savent qu'une émigration aux USA ou en GrandeBretagne signifie presque sûrement un départ définitif. Ils imaginent déjà leur fille se choisir un mari américain, ils imaginent déjà ne jamais connaître leurs petits-enfants et ça leur fait peur ».

\section{Une nouvelle stratégie des jeunes infirmières : le golfe comme tremplin vers l'occident} dernières années développent une nouvelle stratégie dont le but est d'atteindre l'Occident. Les projets de Neejee, une étudiante d'Ernakulam âgée de 20 ans, en sont un exemple significatif :

«Après mon diplôme, je vais travailler ici [en Inde] pendant deux ans. C'est l'expérience minimum demandée dans le Golfe et ça me permettra d'économiser le prix de l'agence de voyage. Mon frère qui travaille en Arabie Saoudite m'aidera aussi parce que les agences demandent de plus en plus cher ${ }^{36}$. J'espère aller plutôt dans les Émirats ou au Koweit parce que c'est mieux payé [que dans d'autres pays du Golfe]. Là-bas, si je travaille pendant deux ans, je pourrai me marier [i.e. qu'elle aura déjà pu économiser suffisamment pour aider à constituer sa dot] et mon mari pourra venir me rejoindre. Après un an ou deux, on pourra avoir des enfants. Pendant ce temps-là, j'aurai eu le temps et l'argent pour préparer le TOEFL et peutêtre le $\mathrm{CGNFS}^{37}$ et nous pourrons partir au Royaume-Uni ou au Connecticut où j'ai de la famille».

Les étudiantes et les jeunes infirmières émigrées que j'ai rencontrées parlent ainsi de leur avenir, d'une façon étonnamment programmée. Celles avec qui je me suis entretenue en Oman ou dans les Émirats avaient d'ores et déjà franchi quelques-unes de ces étapes. Les plus récemment arrivées, célibataires, vivant en foyer, économisaient pratiquement tout leur salaire pour l'envoyer à leurs parents, à l'exception de l'argent nécessaire à l'achat des bijoux en or (moins cher dans le Golfe) qui constitueront leur dot. Celles qui étaient là depuis plus de deux ans étaient soit mariées depuis peu (à trois exceptions près, avec un mari choisi par la famille et pour $60 \%$ d'entre elles avec un homme qui n'avait pas encore émigré); celles qui étaient là depuis plus de trois ou quatre ans avaient réussi à faire venir leur mari et la plupart avaient déjà un enfant (vivant avec elles ou, le plus souvent, au pays) et toutes préparaient les examens nécessaires à l'émigration en Occident avec une idée plus ou moins précise du pays visé. Une dizaine d'entre elles avaient réussi ces examens et se préparaient au départ. Il est évidemment difficile de savoir si ces plans si précisément dressés fonctionnent toujours aussi bien : j'ai ainsi rencontré à Mascate une jeune femme enceinte plus tôt que prévu (soit moins de deux ans avant la fin d'un contrat qui n'autorisait pas de grossesse ce qui a entraîné sa démission). Son époux qui ne croyait pas à un accident a tout d'abord très mal vécu ce contretemps. "Elle est irresponsable. On avait dit qu'on attendrait encore un an ou deux!", m'a-t-il dit, montrant au passage qu'il n'y a guère de tabou concernant la contraception $^{38}$. J'ai retrouvé Mary plus tard chez ses parents au Kerala où elle profitait de la fin de sa grossesse pour préparer activement l'examen de la Commission of

Revue européenne des migrations internationales, vol. 21 - $n^{\circ} 1$ | 2005 
Graduates of Foreign Nursing Schools. Son époux, revenu pour assister à un mariage, semblait avoir accepté la situation, se réjouissant même que cela puisse finalement accélérer leur projet de migration vers l'Occident (pour peu que Mary réussisse tout de suite son examen). Coïncidence ou non, il s'agissait justement de l'un des trois couples rencontrés ayant fait un "mariage d'amour " et n'ayant donc déjà pas tout à fait respecté la norme. Toutefois, dans la plupart des cas, le "programme migratoire " semble bien être suivi d'assez près ou, tout au moins, n'est jamais perdu de vue quels que soient les aléas dus aux limites de contrat, à des parenthèses imprévues entre deux périodes de migration pour raisons familiales ou autre. L'un des résultats en est un net retard de l'âge au mariage des jeunes filles migrantes (plus de 25 ans pour les infirmières migrantes contre 22,6 ans pour l'ensemble des femmes chrétiennes au Kerala - communauté où l'âge au mariage des femmes est déjà le plus élevé - et 20,3 ans pour l'ensemble des femmes malayalies) et donc de l'âge au premier enfant (plus de 27 ans contre 21,9 pour l'ensemble des femmes malayalies) ${ }^{39}$. Les jeunes infirmières sont bien conscientes de cette particularité dont elles sont plutôt fières, allant jusqu'à juger sévèrement la maternité plus précoce comme une preuve de retard culturel et social.

31 On assiste donc à une modification des projets de vie des plus jeunes par rapport aux infirmières émigrées âgées de plus de 35-40 ans. Pour ces dernières, l'émigration dans le Golfe n'a explicitement pour seul but qu'un retour au Kerala dès que les économies réalisées autoriseront l'accomplissement des principaux objectifs : soit, le plus souvent, la construction d'une maison et l'achat des biens d'équipement, l'acquisition d'une voiture, de quoi payer aux enfants des études supérieures, y compris en Occident et, enfin, un minimum de liquidités qui permettent de "voir venir " pendant quelques années. Une fois rentrée au pays, la plupart d'entre elles arrêtent alors de travailler (sauf à trouver un poste d'encadrement ou d'enseignement), marquant aussi de cette manière l'ascension sociale opérée : "Pourquoi s'échiner pour 3000 roupies par mois sans pour autant obtenir le moindre respect le la part des malades ou des médecins? À ce moment-là, autant rester [ou retourner] travailler dans le Golfe! " telle est la réponse habituelle lorsqu'on demande aux ex-émigrées si elles envisagent de retravailler un jour. Travailler à nouveau en Inde en tant que simple infirmière après un passage dans le Golfe est le signe d'un échec ou d'un événement important (maladie ou décès d'un époux, faillite d'une entreprise commerciale, divorce...).

En revanche, pour les plus jeunes, l'émigration dans le Golfe est désormais perçue comme un préliminaire à la " véritable » migration, celle vers l'Occident, les possibilités d'embauche n'ayant cessé d'augmenter ces dernières années : en plus des États-Unis et de la Grande-Bretagne, l'Australie, la Nouvelle-Zélande, le Canada ou même la Suisse, l'Italie et l'Allemagne leur ont ouvert leurs portes. L'information circule rapidement et toute nouvelle opportunité fait aussitôt l'objet de stratégies appropriées : ainsi dès que le Canada a décidé de donner la préférence à des immigrants ayant des notions de français, les infirmières candidates au départ se sont précipitées dans les Alliances Françaises tant en Inde que dans le Golfe ${ }^{40}$. Toutefois, c'est la Grande-Bretagne et les États-Unis qui recrutent en plus grand nombre et ce sont aussi les deux pays les plus prisés par les candidats indiens à l'émigration en général, et donc par les infirmières et leur famille. Au demeurant, les États-Unis visent explicitement un recrutement en Inde comme en témoignent les centres qui se sont ouverts récemment dans diverses villes indiennes pour faire passer l'indispensable test de la CGNFS ou encore la page d'accueil 
du site web de cette même institution qui représente en premier plan une infirmière indienne.

À nouveau, il est très difficile d'évaluer l'autonomie réelle des jeunes femmes dans leur projet migratoire vers l'Occident tant est forte la pression sociale (ainsi qu'elles l'expriment, ce serait presque indécent de ne pas se rendre en Occident lorsque la possibilité se présente, alors que nombre de leurs compatriotes, dont c'est le rêve, n'ont guère de moyens d'y parvenir). Ainsi, lorsqu'elles déclinent les différentes étapes d'un plan de vie qui semble mûrement réfléchi, elles ne manquent jamais d'en pointer les écueils, à savoir, selon elles, la coupure quasi définitive avec leur pays et, surtout, le décalage entre leur culture et celle qu'auront probablement leurs enfants. « Mais c'est la vie!», concluent-elles généralement, comme s'il n'y avait pas vraiment de choix. On rencontre aussi des jeunes femmes dont le discours contredit cet apparent fatalisme : elles expliquent alors clairement leur projet de migration comme un bon moyen de se soustraire au poids de la famille traditionnelle. Elles ne sont guère à être aussi explicite, même si celles qui le sont affirment être de plus en plus nombreuses à envisager la migration sous cet angle (ou prétendent «être simplement plus franches » à cet égard que leurs collègues). Pour elles, le passage à l'Occident est alors envisagé comme une libération qui leur permettra de vivre plus facilement leurs aspirations individuelles: "Je ne veux pas travailler toute ma vie pour entretenir toute une pléthore d'oncles, de tantes et de cousins, pour payer la dot d'Unetelle ou les études d'Untel [...] Je n'ai pas l'intention de revenir m'installer au Kerala juste pour montrer comme j'ai bien réussi en construisant une maison de trois étages dans laquelle je passerais le reste de mon temps à recevoir les femmes de la famille ou de la paroisse pendant que mon mari passerait son temps à véhiculer les uns ou les autres dans sa Toyota de luxe" m'a ainsi expliqué Alice, une jeune femme de 32 ans qui s'apprêtait à quitter Dubaï avec son époux et ses deux enfants après un séjour de six ans, pour aller aux États-Unis où elle venait d'obtenir un contrat. Cette jeune femme avait pourtant fait un mariage arrangé, tradition qu'elle ne remettait nullement en cause au demeurant, critiquant seulement la pratique de la dot. De fait, la plupart des entretiens que j'ai eus avec des infirmières rentrées au Kerala font bien ressortir une difficulté à s'y réintégrer. Cette difficulté s'exprime à plusieurs niveaux dans la relation avec la famille au sens large, mais aussi dans la nostalgie du cosmopolitisme ou de l'anonymat qui l'accompagne (le Kerala est alors ressenti comme trop « provincial » en quelque sorte).

Aujourd'hui, les pays du Golfe n'apparaissent ainsi plus comme la destination en soi de l'émigration des infirmières indiennes, du moins telle n'est plus la façon dont elles l'envisagent. Cette destination reste pourtant une sorte de passage obligé ${ }^{41}$. Tout d'abord parce qu'elles peuvent y partir avec leur seul diplôme indien et, donc, plus rapidement et plus aisément. Les diplômes exigés pour une émigration en Occident anglophone (diplôme de langue anglaise nécessaire presque partout et test de compétence pour les USA) demandent, en effet, un investissement coûteux en temps et en argent puisqu'il est presque obligatoire de suivre des cours de préparation. Le test de la CGFNS américaine est, en outre, assez difficile à réussir puisque, d'après les sources officieuses que j'ai pu obtenir auprès de directrices d'écoles, seules $17 \%$ des candidates indiennes y parviendraient chaque année. On ne gagne donc pas l'Occident si facilement. Enfin les « agences de voyage » fournissant les contrats font payer deux à trois fois plus cher pour l'Occident que pour les pays du Golfe. 
Mais, cette nouvelle façon de considérer la migration dans le Golfe comme un préalable à une autre migration entraîne une évolution dans la façon dont les infirmières indiennes considèrent ces pays. Leur jugement n'en devient que plus sévère, la critique plus acerbe, d'autant qu'ils n'offrent plus les meilleurs revenus ${ }^{42}$. Le temps passé dans le Golfe est dès lors vécu comme une sorte de pénible prélude à l'entrée dans le "véritable " avenir ("Mon véritable avenir n'est pas ici!", m’ont ainsi affirmé plusieurs jeunes infirmières en Oman ou dans les Émirats). Pourtant ce séjour dans le Golfe fait bien fonction d'initiation à la vie en émigration. Car, de façon qui peut paraître paradoxale, la migration dans le Golfe est aussi décrite comme une transition en douceur, un genre de sas. Parce qu'il n'y a pas de vrais contacts avec la population autochtone, pas plus qu'entre les différentes communautés émigrées, c'est essentiellement un entre-soi que vivent les infirmières. Ce n'est plus vivre au pays, mais c'est toujours vivre entre Malayalis dès que le travail est fini. La ségrégation qui prévaut dans le Golfe évite de se poser des questions sur son identité, sa culture, son style de vie (ou du moins permet-elle de les résoudre de façon simple), à la différence de la migration en Occident où les problèmes de l'adaptation, de l'intégration ou de l'acculturation se posent très consciemment pour toutes les femmes que j'ai rencontrées : pour résumer leur sentiment, on pourrait dire que si la vie en Occident peut se faire en partie au sein de la diaspora indienne présente quasiment partout, on n'échappe jamais cependant - et on ne souhaite d'ailleurs pas échapper - à un contact avec les autochtones (notamment avec les enfants qui apprendront, voire en prendront, la langue); ce contact est ressenti en grande partie comme positif dans la mesure où, à la différence de ce qu'ils ressentent pour les habitants du Golfe, les Indiens ont un respect certain pour la culture occidentale, même s'ils n'adhèrent pas à toutes ses valeurs. La migration dans le Golfe se réduit alors à apprendre à vivre loin de ses proches et à travailler dans un milieu cosmopolite, ce qui peut être difficile, mais peutêtre moins angoissant qu'une confrontation réelle - même souhaitée - avec une autre culture. En outre, pour les infirmières, l'exercice de leur métier est, aux dires de toutes, finalement plus facile dans le Golfe qu'en Inde, en raison de meilleures conditions de travail mais aussi d'un plus grand respect que leur témoigneraient ici médecins et patients ${ }^{43}$ (peut-être en partie parce que la notion d'« impureté » n'a pas dans ces pays le même poids qu'en Inde).

Tous ces éléments expliquent sans doute un fait qui paraît surprenant a priori: le manque apparent d'inquiétude et même l'assurance avec laquelle les jeunes infirmières indiennes partent pour le Golfe, seules en général en un premier temps, et la façon dont elles se débrouillent, bien qu'elles aient vécu toute leur enfance et adolescence d'une façon plus que protégée au sein de la famille à l'instar de la plupart des jeunes filles indiennes. Cela est vraisemblablement dû en partie au fait que l'émigration fait aujourd'hui partie intégrante de la culture du Kerala, d'où la capacité à adopter aussi rapidement dans les projets les nouvelles opportunités dans tel ou tel pays ou à envisager une nouvelle migration après un ou plusieurs retours au pays. Si l'émigration reste aventureuse pour les plus pauvres, sans instruction et sans qualification, à la merci de toutes les exploitations, elle est en revanche devenue un parcours presque banal pour les moins démunis. Dans le cas des infirmières, elle est aussi une voie vers un nouveau statut social et un autre statut en tant que femme. 


\section{Des conséquences sur les relations au sein de la famille}

37 Si une migration massive des hommes conduit à une autonomie plus grande pour les épouses restées au pays comme le montre Leela Gulati pour le cas du Kerala (Gulati, 1993), les changements sont encore plus flagrants lorsque les femmes sont elles-mêmes les têtes de pont de cette migration. Ainsi, les infirmières migrantes sont bien conscientes d'endosser un rôle traditionnellement vu comme masculin lorsqu'elles procurent à la famille une large partie de ses revenus et, pour celles qui partent avant leur mariage, lorsqu'elles participent amplement à la constitution de leur dot (rôle classiquement dévolu au père et aux frères); en revanche, elles ne soulignent plus guère leur office de passeport pour la migration des membres de la belle-famille - à commencer par l'époux - tant il est évident pour elles, qu'aujourd'hui, c'est bien pour cela qu'on devient infirmière ${ }^{44}$. Cependant si toutes se disent fières de leur apport à la famille (qu'il faut toujours dans le contexte indien entendre au sens large quand bien même le couple et ses enfants ont leur propre logement), leurs propos sont souvent beaucoup plus mitigés, tout spécialement lorsqu'on aborde la situation économique familiale après quelques années passées à l'étranger : lorsque d'autres membres de la famille (époux et beaux-frères) ont eux-mêmes bien réussi financièrement au pays ou en migration, leur contribution au budget familial leur semble juste et utile. Mais que les hommes aient été incapables de faire suffisamment prospérer l'argent économisé (voire, ce qui arrive souvent, l'aient perdu dans des entreprises hasardeuses), qu'ils soient incapables de trouver un travail correct ou de le conserver et que, de surcroît, les belles-sœurs restent femme au foyer "élevant tranquillement les enfants " et les migrantes se sentent alors flouées. Elles disent notamment leur frustration de «sacrifier» (ou d'avoir dû sacrifier) pour rien, la relation avec leurs enfants et formulent alors des récriminations sur ce qu'elles vivent comme un détournement affectif ("J'ai laissé ma fille lorsqu'elle avait un an, maintenant même si elle m'appelle maman, c'est vers ma belle-sour qu'elle court si elle pleure ou se blesse ", dit ainsi Sara qui soupçonne sa belle-sœur, "malgré tous les cadeaux qu'elle lui a fait ", de chercher à détourner sa fille de six ans de l'affection maternelle). Dans presque tous les cas, lors des retours au pays, les infirmières migrantes estiment que la famille intervient trop dans l'éducation de leurs enfants et dans les affaires du couple, dispose trop facilement de l'argent qu'elles gagnent et accusent régulièrement leur époux d'être trop généreux ou de ne pas savoir dire non: ainsi plus leur contribution au budget familial est importante, moins le contrôle par d'autres de cet argent est tolérable et il devient quasiment insupportable si, après quelques années, le succès économique et social n'est pas au rendez-vous lors de leur retour - temporaire ou définitif - au foyer. Mais on repère bien cependant un changement d'attitudes au sein de la famille des migrantes, à commencer par une différence de statut entre ces dernières et leurs belles-sœurs «femmes au foyer » et une relation différente à la belle-mère. L'anecdote suivante en témoigne : à de nombreuses reprises, lors d'entretiens que j'avais avec des infirmières (migrantes en congé ou ex-migrantes) au logis familial, celles-ci commandaient avec autorité à une belle-sœur et même à la belle-mère de nous préparer et de nous servir thé ou rafraîchissement : inversement radical des rôles en Inde où une belle-fille est censée servir sa belle-mère. Il semble aussi que l'époux soit de plus en plus appelé à appuyer sa femme lorsqu'il est question d'argent qu'elle estime en droit désormais de 
verser au moins occasionnellement à sa propre parentèle, ce qui est là encore contraire à la tradition indienne. Ces femmes paraissent également en mesure de peser sur la scolarité de leurs enfants notamment en bloquant les fonds nécessaires pour leurs études supérieures. Leur pouvoir n'est, en revanche, pas suffisant pour obtenir un droit de veto concernant, par exemple, l'investissement des économies dans une entreprise commerciale (ce qu'elles considèrent comme "une affaire d'hommes»), mais elles n'hésitent pas à formuler des critiques virulentes en présence même de leur époux en cas d'échec. En réalité, ce que les migrantes ou ex-migrantes ont désormais bien du mal à supporter, c'est le cadre même de la famille élargie et ses contraintes, aussi bien dans les gestes de la vie quotidienne que dans la redistribution des richesses ${ }^{45}$ (à la différence de femmes de milieu beaucoup plus pauvre que j'ai rencontrées et qui continuent à valoriser ce qu'elles voient - elles - comme une indispensable solidarité familiale). L'aspiration à vivre en famille nucléaire est ainsi partagée par presque toutes les migrantes et par les jeunes filles candidates à la migration en sachant que la migration est un excellent moyen d'y parvenir alors qu'il est financièrement et légalement compliqué de recréer une maisonnée élargie particulièrement dans les pays du Golfe.

C'est l'expérience de leurs aînées qui permet aux plus jeunes de bâtir leurs plans d'avenir et leur stratégie. Ainsi se marier alors qu'on travaille déjà à l'étranger implique, le plus souvent, que le jeune couple échappe - au moins pendant quelques années - à la vie communautaire : "Lorsque tu vis avec ton mari seulement et qu'il n'y a pas autour sa mère, ses frères, enfin tous les beaux-parents et même tous les voisins, il est moins influencé, il a moins peur de passer pour un lâche devant sa femme et il y a beaucoup plus d'égalité [...] On apprend aussi à mieux se connaître et à mieux se comprendre [...] Pour l'avenir des enfants aussi c'est beaucoup mieux parce qu'on ne disperse pas l'argent à droite et à gauche » expose ainsi Neejee, jeune célibataire, dont les deux sœurs sont également infirmières, l'une à Dubaï et l'autre en Australie. Elle me dit aussi que ses sœurs disposent d'un compte commun avec leur époux - ce que m'ont pareillement assuré nombre d'infirmières dans le Golfe - et plaisante à ce sujet : "Cela vaut mieux pour eux puisque mes sœurs gagnent plus qu'eux!» (à la question "Qui gère l'argent? ", la réponse est alors presque invariablement : «On gère en commun, mais il faut faire attention à ne pas froisser la susceptibilité de son mari parce qu'un homme n'a pas l'habitude de gagner moins que sa femme $»^{46}$ ). L'expérience apprend aussi à ces jeunes femmes que, même si lors du retour au pays, la norme est de plus en plus la vie en famille restreinte, ce qui se veut aussi signe de modernité (et je n'ai effectivement rencontré aucun exemple, dans le cas des infirmières, de construction de maison destinée a priori à la famille élargie), il n'en reste pas moins que la pression familiale et celle du voisinage s'affirment à nouveau de façon trop forte selon elles. C'est à cette occasion que s'exprime alors l'attrait du cosmopolitisme et du relatif anonymat qu'offre la vie à l'étranger. De nombreuses remarques m'ont ainsi été faites sur la plus grande liberté tant sur la façon de cuisiner, de se vêtir, de se maquiller, de se comporter en public avec son mari (à l'exception de l'Arabie Saoudite en ce qui concerne ces derniers aspects) que de recevoir des amis et d'avoir des activités ou des loisirs à l'extérieur de la maison. Ce désir de plus d'autonomie ne les amène cependant pas à remettre en cause le mariage arrangé ni à rejeter le système de la dot. Il n'y a là rien de paradoxal. En ce qui concerne la dot, c'est en la finançant en grande partie qu'elles commencent par s'affranchir de leur père et frères, tout en offrant du prestige à la famille grâce au mari ainsi «gagné ». En ce qui concerne le mariage, et comme le montrent aussi les pratiques de la bourgeoisie indienne la plus cosmopolite (y compris des jeunes nés et élevés à l'étranger), la vision 
occidentale de la "supériorité » du "mariage d'amour », ne convainc toujours pas les Indiens et les Indiennes.

\section{Conclusion}

Mais un paradoxe demeure. Le soutien de la famille élargie est presque toujours indispensable pour financer les études, puis les frais d'émigration de ces jeunes infirmières. Ce soutien est également nécessaire pour prendre soin des enfants laissés au pays lors du séjour dans le Golfe. Or plus le temps de la migration est long ou plus il se renouvelle, moins les infirmières sont prêtes à reprendre place, physiquement mais aussi financièrement, dans cette famille traditionnelle. Elles ont maintenant acquis une bonne réputation sur le marché matrimonial, et en tant qu'infirmière, un meilleur statut au sein de leur communauté (et dans la société malayalie) en raison de leur capacité à avoir de bons revenus et à fournir le visa convoité à d'autres membres de la belle-famille (y compris, aujourd'hui la fameuse Green Card), mais leurs aspirations tendent vers un mode de vie beaucoup plus individualiste, en famille nucléaire autant que possible pour commencer. Ces aspirations et la stratégie susceptible de les combler sont nées peu à peu, au fil de l'expérience acquise au cours de ces trente années d'émigration des infirmières indiennes dans le Golfe. Devenir infirmière aujourd'hui au Kerala représente plus qu'un passeport pour le monde - que les plus jeunes sont au demeurant particulièrement désireuses de découvrir -, c'est aussi un passeport pour une vie plus indépendante et plus autonome puisque, au moins dans leur milieu social, cela s'avère plus facile à construire à l'étranger que dans leur propre pays, surtout avec l'Occident en ligne de mire. À la différence de leurs aînées, les jeunes infirmières ou futures infirmières n'envisagent donc plus la migration comme une simple parenthèse dans leur vie, mais comme une chance d'échapper à un futur trop conventionnel et trop prévisible.

\section{BIBLIOGRAPHIE}

BENEÏ Véronique (1996) La dot en Inde. Un fléau social ? Socio-anthropologie du mariage au Maharashtra, Paris, Karthala/Institut Français de Pondichéry, 291 p.

DUMONT Louis (1966) Homo Hierarchicus, le système des castes et ses implications, Paris, Gallimard, coll. Tel, $449 \mathrm{p}$.

GEORGE Sheba (2000) « Dirty Nurses » and « Men who play »: Gender and Class in Transnational Migration, in Michael Burawoy Ed., Global Ethnography : forces, connections and imaginations in a postmodern world, Berkeley, University of California Press, pp. 144-74.

GULATI Leela (1993) In the Absence of their Men, the impact of male Migration onWomen, New Delhi, Sage Publication.

JEFFREY Robin (1993) Politics, Women and well-being, How Kerala became amodel, New Delhi, Oxford University Press (Paperback), 285 p. 
LONGVA Ahn Nga (1997) Walls built on sand. Migration, exclusion and society inKoweit, Oxford, Westview Press, 266 p.

MENSKI Werner (Ed) (1998) South Asians and the Dowry Problem, Londres, Trentham Books Ltd. MOHAN N. Shantha (1990) Status of nurses in India, New Delhi, Uppal Publishing House, 114 p. OSELLA Filippo, OSELLA Caroline (2000) Social mobility in Kerala, Modernityand identity in conflict, London, Pluto Press, 320 p.

PRAKASH B.A. et al (1994) Kerala's economy : performance, problems, prospects, New Delhi, Sage.

SARADAMONI K. (1999) Matriliny Transformed. Family, Law and Ideology in Twentieth Century Travancore, New Delhi, Sage Publications and Altamira Press, 176 p.

SEKHER T.V. (1997) Migration and social change, New Delhi, Rawat Publication, 182 p.

TARABOUT Gilles (1997) « Une configuration sociale régionale : le Kerala », Historiens et Géographes, 356, pp. 243-56.

THEAU Benoît, VENIER Philippe (2001) Kerala : la force de l'ambition, Poitiers, Orcade, $169 \mathrm{p}$.

VISVANATHAN Susan (1993) The Christians of Kerala. History, Belief and Ritual among the Yacoba, New Delhi, Oxford University Press, 279 p.

ZACHARIAH K.C., KANNAN K.P., IRUDAYA RAJAN S. (2000) Migration inKerala State, India: dimensions, determinants and consequences, working paper II, Centre for Development Studies, Thiruvananthapuram, Indo-Dutch Program on Alternatives in Development, 240 p.

ZACHARIAH K.C., KANNAN K.P., IRUDAYA RAJAN S. (2002) Kerala's Gulf connection. CDS studies on international labour. Migration from Kerala State in India, Trivandrum, Centre for Development Studies, $230 \mathrm{p}$.

ZACHARIAH K.C., MATHEW E.T., IRUDAYA RAJAN S. (1999) Impact of migration on Kerala's economy and society, Working paper $n^{\circ}$ 297, Trivandrum, Centre for Development Studies, 43 p.

ZACHARIAH K.C., MATHEW E.T., IRUDAYA RAJAN S. (2004) Dynamics of Migration in Kerala : Dimensions, Differentials and Consequences, New Delhi, Orient Longman, $476 \mathrm{p}$.

\section{NOTES}

1. Cet article présente les premiers résultats d'une recherche entreprise en 2002. Elle est basée sur un terrain de deux fois trois mois en Inde (au Kerala et à Bombay) et de six semaines en Oman ainsi qu'aux Émirats Arabes Unis. J'ai interrogé un peu plus de deux cents infirmières ou futures infirmières : 80 infirmières émigrées dans le Golfe, une soixantaine d'infirmières ex-émigrées vivant au Kerala et autant d'étudiantes en école d'infirmières du Kerala et de Bombay. Les entretiens ont été menés individuellement auprès de 72 de ces femmes, les autres ont été collectifs (jusqu'à cinq personnes lors d'un même entretien). J'ai aussi interrogé douze surveillantes générales d'hôpitaux (non indiennes) à Oman et aux Émirats, dix surveillantes générales (n'ayant jamais émigré) et quatre directeurs du personnel d'hôpitaux au Kerala, ainsi que quinze directrices d'école d'infirmière au Kerala et à Bombay. Quatre prêtres catholiques malayalis ont répondu à mes questions (dont un à Mascate). J'ai enfin été invitée, au Kerala, dans dix-sept familles d'étudiantes ou d'infirmières émigrées pour des périodes allant de deux à six jours. Nota : tous les prénoms utilisés dans ce texte sont fictifs. Je tiens à remercier Eliane Daphy et Agnès Jeanjean ainsi que les coordinatrices du numéro pour la relecture de cet article. 
2. Le personnel infirmier est aussi constitué de Philippines. Elles sont cinq à six fois moins nombreuses que les Indiennes d'après les observations que j'ai pu faire moi-même dans les quinze hôpitaux d'Oman et des Émirats que j'ai visités.

3. Cette formation existe aujourd'hui dans la plupart des pays du Golfe, mais les candidates y sont peu nombreuses. De plus, selon les médecins ou infirmières générales rencontrés, leur formation est beaucoup trop scolaire et leur faible niveau d'anglais est un gros handicap dans leurs échanges avec le personnel médical.

4. Il n'existe pas de statistiques vraiment fiables concernant le nombre d'infirmières indiennes émigrées. Toutefois si l'on recoupe le nombre d'infirmières par habitants dans les différents pays du Golfe et le pourcentage d'infirmières indiennes dans les différents hôpitaux que j'ai visités, ce chiffre est vraisemblable.

5. Notons qu'en raison du nombre important d'infirmières formées, l'Inde ne semble pas encore menacée de pénurie dans ce secteur.

6. Recensement de 2001, cf. Zachariah et al., 2004, p. 16.

7. Les habitants du Kerala sont appelés «Malayalis» d'après le nom de leur langue, le malayalam.

8. Chiffres pour 2001.

9. Chiffres pour 1999.

10. Dont un certain nombre d'infirmières qui sont aux USA depuis déjà longtemps (George, 2000) ou en Grande-Bretagne, mais le recrutement massif vers ces pays est récent.

11. Tandis que les «syrians" se considèrent comme relativement bien situés dans la hiérarchie des castes (équivalents au Vaishyas, voire plus), les conversions dues aux colonisations portugaise et britannique ont principalement concerné les communautés d'intouchables. Ces derniers, chrétiens catholiques dits « latins » et chrétiens protestants représentent aujourd'hui la moitié des Chrétiens, soit $10 \%$ de la population keralaise.

12. Essentiellement les catholiques «syrians" (pour $70 \%$ des infirmières rencontrées contre $30 \%$ de « Latin Roman » catholiques).

13. Les infirmières citent d'ailleurs souvent Florence Nightingale et surtout Mère Teresa comme sources d'inspiration.

14. Je n'ai pu, jusqu'à présent, trouver les raisons pour lesquelles les femmes chrétiennes d'autres régions de l'Inde n'avaient pas investi ce créneau professionnel : je pense en particulier à Goa où les Chrétiens sont aussi nombreux.

15. 1 acre $=0,6$ ha.

16. Il s'agissait là de familles dont aucun membre n'avait encore émigré à l'étranger. Lorsqu'un enfant au moins a émigré, on voit apparaître d'autres biens de consommation: meubles à l'occidentale, magnétoscope, divers appareils ménagers, parfois, une voiture.

17. Cela vaut pour l'ensemble de la fratrie.

18. 50 Roupies $=1$ Euro.

19. Pour une étude plus approfondie du sujet et de ses conséquences tragiques aujourd'hui (en particulier infanticides, avortements ou jeunes épouses brûlées vives, mais aussi ruine des familles où les filles sont plus nombreuses que les garçons), voir par exemple Beneï, 1996 ou Menski, 1998.

20. Ma traduction comme pour toutes les citations de ce texte.

21. De la même manière qu'en Inde, les hôpitaux gouvernementaux sont ceux qui payent le mieux parmi les hôpitaux du Golfe. Sa jeune femme gagnait ainsi 300 Omani Ryals (1 O.R. = 3 Euros environ).

22. Annonces parues en juin 2003 sur le site http://www.keralamatrimony.com/

23. Selon les infirmières concernées et leur famille, c'est en grande partie une jalousie, pour les opportunités migratoires et les revenus des infirmières émigrées, qui explique ces rumeurs ou ces propos malveillants. 
24. Environ $7 \%$ d'Hindoues et $3 \%$ de Musulmanes pour les écoles que j'ai visitées en Inde. Notons que les Musulmanes auraient encore plus de chance de trouver un bon poste dans le Golfe, les annonces de travail précisant souvent « Musulmane de préférence ».

25. Au Kerala même, la pression à l'émigration est suffisamment forte pour que les hôpitaux publics accordent désormais jusqu'à 15 ans de disponibilité (en trois périodes de 5 ans maximum).

26. Trois de mes amies indiennes sont parties à l'étranger pour une année ou deux d'enseignement dans une université alors même qu'elles avaient des enfants en bas âge. Elles m'ont fait remarquer qu'elles avaient le sentiment d'échapper au sentiment de culpabilité d'avoir « laissé » leurs enfants derrière elles, contrairement, leur semblait-il, à des collègues occidentales dans la même situation.

27. Les gens fortunés du Golfe continuent d'ailleurs à se faire soigner à l'étranger, particulièrement en Inde à Bombay. Un hôpital luxueux (en équipement médical mais aussi «hôtellerie ») vient aussi de s'ouvrir à Cochin au Kerala dont les fonds viennent en partie des Émirats. Il a été inauguré durant l'hiver 2002 en présence du Ministre de la Santé des Émirats Arabes Unis.

28. Capitale du Kerala. Les nombreux commerces « indiens » des pays du Golfe n'appartiennent pas en propre aux Indiens puisque les étrangers n'ont pas le droit dans ces pays de devenir propriétaire. Ainsi, quand bien même les immigrants amènent la plus grosse part du capital - voire la totalité du capital - nécessaire à l'ouverture d'un commerce, il doit exister un «sponsor» autochtone, propriétaire en titre et qui percevra des dividendes sur ledit commerce. Toutefois un «coup d'œil » extérieur ne permet pas de saisir ce système dans la mesure où le propriétaire autochtone n'est pratiquement jamais présent et qu'il n'intervient pas non plus dans l'agencement du magasin.

29. Concurrence qui ne me paraît pas réelle mais qui repose sur des a priori des Indiennes selon lesquels les Philippines auraient plus de chances, vu la « vague islamique », puisqu'elles sont pour beaucoup musulmanes, et aussi - paradoxalement - parce qu'elles "auraient la réputation d'être des femmes aux mœurs beaucoup plus légères » (sous-entendu de «bonnes affaires » pour les administratifs autochtones ou les médecins) que les Indiennes. Toutefois, on ne peut parler de réels antagonismes entre Indiennes et Philippines, les amitiés nouées sont nombreuses et les Indiennes vantent très souvent, par ailleurs, le "sens de la fête » que posséderaient les Philippines. Nota : Je n'ai trouvé aucune recherche concernant les 30 infirmières philippines dans le Golfe. Des discussions que j'ai eues avec quelques-unes d'entre elles font déjà apparaître des différences notables avec leurs collègues indiennes: tout d'abord le métier d'infirmière serait depuis toujours un métier valorisé aux Philippines, ensuite les stratégies migratoires ne se recouperaient pas puisqu'il semblerait que les Philippines présentes dans le Golfe ne jouent pas ce rôle de tête de pont migratoire (restant beaucoup plus souvent seules que les Indiennes), enfin l'émigration dans les pays du Golfe serait un vrai pis-aller, les États-Unis étant la destination jugée « naturelle».

30. Cette précarité n'est pas spécifique aux infirmières ou même aux femmes émigrées dans le Golfe, les hommes émigrés la subissent de la même manière. Pour une explication de la législation concernant les immigrants dans le Golfe, et en particulier du système du «sponsoring ", voir Longwa, 1997, qui détaille l'exemple du Koweit.

31. À l'exception notable de l'Arabie Saoudite où il n'existe, en particulier, pas de liberté de culte. Plusieurs femmes, ex-émigrées en Arabie Saoudite, m'ont raconté comment elles se réunissaient en cachette pour des séances de prière ou pour fêter Noël ou Pâques, comment elles étaient fouillées par les douaniers à la recherche de bible ou de cassettes de cantiques. Cette interdiction de pratiquer est ressentie comme particulièrement dure par les Malayalies chrétiennes généralement très religieuses et comme une grave atteinte à leur liberté. Toutes ont employé les mêmes termes : « En Arabie Saoudite, pour nous c'est la prison », faisant allusion à la fois 
à l'obligation de se voiler, à l'interdiction de sortir non accompagnée d'un homme et surtout à l'interdiction de prier.

32. Parce que leur mari avait un bon travail en Inde, parce qu'il était malade, etc.

33. Il est assez aisé d'avoir un « visiting visa » pour tous les pays du Golfe dès lors qu'un conjoint y travaille légalement. Entre Oman et les Émirats, les immigrés ont seulement besoin d'une autorisation assez simple à obtenir pour passer d'un pays à l'autre.

34. En Inde, les enfants sont traditionnellement considérés comme faisant partie de la famille du mari. Au Kerala, la tradition matrilinéaire qui a prévalu jusqu'au début du XXe siècle les rattachait au contraire à la famille de la femme, mais il ne semble pas que cette tradition ait laissé de trace, chez les Chrétiens tout au moins, en ce qui concerne la garde des enfants, comme en témoigne la priorité donnée à la belle-famille.

35. Il n'est pas possible de trouver d'employées de maison sur le marché local du travail. Les familles indiennes font donc souvent venir des jeunes femmes de leur pays à qui elles payent un salaire assez dérisoire (moins de 100 Euros, aux dires d'interlocuteurs émigrés en Oman, euxmêmes employeurs). Il faut cependant payer le voyage de l'employée, mais surtout lui obtenir un visa, ce qui semble être devenu difficile pour les émigrés indiens.

36. Les " agences de voyage ", qui fournissent le contrat de travail et s'occupent d'obtenir le visa, demandent l'équivalent de trois mois du salaire offert dans le Golfe plus les frais de voyage et de visa. Soit, actuellement, un minimum de cent mille roupies.

37. TOEFL: Test of English as Foreign Language. CGNFS : examen organisé par la Commission of Graduates of Foreign Nursing Schools (organisme américain). Le premier diplôme est nécessaire pour émigrer dans les pays occidentaux anglophones. Le second est exigé pour travailler comme infirmière aux USA.

38. Le faible taux de natalité au Kerala témoigne d'une pratique massive de la contraception. De plus à ce jour, $67 \%$ des femmes malayalies ont eu recours à la stérilisation après la naissance de leur deuxième enfant. Une pratique que l'Église du Kerala condamnerait, selon mes interlocutrices, alors qu'elle admettrait la contraception (information que je n'ai pu vérifier). Mais l'interdiction de la stérilisation ne paraît pas très respectée malgré la grande religiosité des Chrétiennes malayalies : «Ce ne sont pas eux (les membres du clergé) qui élèvent les enfants! » est le commentaire que j'ai entendu à ce sujet. Il est à noter aussi que cette "interdiction de grossesse » stipulée dans de nombreux contrats d'infirmières dans le Golfe n'est pas vraiment ressentie par ces femmes comme une atteinte à leur liberté dans la mesure où elle correspond généralement à leur propre plan de vie.

39. Source : International Institute for Population Sciences, 2001. National Family Health Survey India, 1998-99, Kerala, NFHS-2. Mumbai.

40. Le directeur d'un Centre Français dans le Golfe raconte qu'il avait eu dans les mêmes temps la visite d'un Indien « agent de voyage » qui lui promettait de nombreuses étudiantes pour peu qu'il lui consente un "prix de gros »... De fait, ces nouvelles étudiantes, peu intéressées réellement par la culture française, semblent plutôt embarrasser les enseignants et les étudiants traditionnels des Alliances.

41. Une étude très récente (2003-2004) menée auprès de 10000 foyers du Kerala par le Centre for Development Studies de Trivandrum (non encore publiée) montre, contre toute prévision, que le nombre de Malayalis dans le Golfe a encore augmenté ces cinq dernières années. Je remercie $\mathrm{S}$. Irudaya Rajan de m'avoir communiqué cette information.

42. Alors que ce constat est fait, certains émigrés indiens font cependant remarquer qu'on y économise plus dans la mesure où on dépense moins puisqu'on ne peut investir dans quoi que ce soit.

43. Et ce malgré le mépris qu'elles disent ressentir de la part des indigènes dès lors qu'elles ne sont plus « en service». 
44. Au grand dam des Principales d'école d'infirmière, qui voient cet objectif comme prenant franchement le pas sur la "vocation » à soigner des malades. Je n'ai pas travaillé assez sur la relation infirmière/malade ni au Kerala, ni dans le Golfe pour pouvoir traiter ici de cette question.

45. À l'exception des soins ou de l'aide dus aux parents (pères et mères) âgés.

46. Lors d'une discussion, à Abu Dhabi, avec un jeune couple dont l'époux gagnait deux fois moins que sa femme, cette dernière s'est empressée de préciser «Mais c'est lui qui paye tout ici; mon salaire, c'est pour les économies ».

\section{RÉSUMÉS}

Lorsqu'à la fin des années soixante-dix, des infirmières commencèrent à être recrutées en Inde pour les hôpitaux des pays du Golfe, ce fut une opportunité inattendue pour les plus aventureuses d'entre elles de s'assurer de bien meilleurs revenus que dans leur propre pays. Une génération plus tard, des dizaines de milliers de jeunes filles, principalement des chrétiennes du Kérala, choisissent expressément ce métier afin d'émigrer dès l'obtention de leur diplôme. Ce diplôme est ainsi devenu un véritable passeport pour l'émigration, pour les infirmières ellesmêmes et, par contrecoup, pour leur proches.

Pour les plus âgées des infirmières, la migration dans le Golfe permet de s'assurer un avenir plus prospère une fois revenues au pays; pour les plus jeunes, cette migration est vue comme une étape avant une migration vers l'Occident. Dans tous les cas, les familles encouragent filles ou épouses à partir pour le Golfe en raison des bénéfices immédiats qu'elles en retirent mais aussi parce que cela s'inscrit dans leurs stratégies d'ascension sociale.

\section{The Emigrant Indian Nurses in the Gulf: From opportunity to Strategy : Haitian Transnational Family.}

When in the middle of the seventies, Indian nurses started to be hired for newly built hospitals in the Gulf, it was an unexpected opportunity for the most adventurous of them to ensure unexpected good wages. One generation later, thousands of young girls, predominantly Christians from Kerala, fill up the nursing schools all over India with the objective of migrating after graduation. Hence the nursing diploma is obviously considered as a passport opening the world not only to the nurse herself, but also to her relatives.

Migration to the Gulf is now considered as an intermediate step before further migration to the West or before to come back in India as housewife. It allows married women to improve widely the financial position of their parents and in-laws remaining in India ; it allows the young single nurses to save for their dowry or to find a husband with a better social background. In either case, families encourage their migration because it is very consciously regarded as a privileged opportunity to increase social mobility.

Las enfermeras indias inmigrantes en los países del Golfo: de la oportunidad a la estrategia.

Al final de los años 1970, cuando se contrataron enfermeras en India, destinadas a los hospitales de los países del Golfo, fue una oportunidad inesperada para las mas atrevidas de ellas que querian obtener mejores sueldos que en su proprio país. Una generación despues, decenas de miles de mujeres jóvenes y principalmente cristianas del Kerala, decidieron deliberadamente de 
aprender el oficio de enfermera para poder emigrar cuando obtenian su diploma. Este se convirtio entonces en un verdadero pasaporte para emigrar, para las enfermeras y, por supuesto, para sus familiares.

Para las enfermeras las mas mayores, la migración hacia el Golfo permitia de asegurarse un futuro mas prospero cuando volvian a su país ; para las mas jóvenes esta migración se concibe como un primer paso antes de una migración hacia el Occidente. En todo los casos, las familias incitan las hijas y esposas a irse hacia el Golfo en razón de los beneficios inmediatos que le sacan pero también porque ese proceso se inscribe en una estratégica de ascención sociale.

\section{INDEX}

Index géographique : Golfe persique

Mots-clés : stratégie migratoire, femmes, causes des migrations, Indiens

\section{AUTEUR}

\section{MARIE PERCOT}

Anthropologue, chercheure au Laboratoire d'Anthropologie Urbaine (UPR O34/CNRS) 